\title{
Hyper-inflammation after COVID-19 mARN vaccination: at the crossroads of multisystem inflammatory disease and adult-onset Still's disease. Does terminology matter?
}

\author{
CRISTIAN BAICUS, CATERINA DELCEA, LARISA PINTE, GHEORGHE ANDREI DAN \\ Carol Davila University of Medicine and Pharmacy, Department of Internal Medicine and Cardiology, \\ Colentina University Hospital, 19 - 21 Stefan cel Mare Blvd., 020125 Bucharest, Romania
}

Key words: COVID-19 vaccine, multisystemic inflammatory syndrome, adult-onset Still's disease, cytokine storm, anakinra.

\section{Dear Editor,}

As the pandemic evolves, different facets of the SARS-CoV-2 infection, as well as immunization, with varying complexity and prognostic implications are discovered. One of them is the multisystem inflammatory syndrome (MIS)[1]. Characterized by elevated ferritin levels and hyper-inflammation with vital implications, MIS was proposed as the fifth clinical entity to constitute the "hyperferritinaemic syndromes", alongside the macrophage activation syndrome (MAS), adult-onset Still's disease (AOSD), catastrophic anti-phospholipid syndrome and septic shock [2]. Although most reports were related to SARS-COV-2 infection, MIS was recently described in relation to COVID-19 vaccination as well [3].

We hereby report the case of a previously healthy 22-year-old male, who received the first dose of BNT162b2 vaccine on May $1^{\text {st }} 2021$. Thirteen days after vaccination, he developed a hyperinflammatory state, fulfilling the criteria for adult multisystem inflammatory syndrome (MIS-A) [2] (fever, sore throat, myalgias, myocarditis, hepatic injury, maculo-papular rash, diarrhoea, hypotension, and highly elevated inflammatory markers including a procalcitonin level of $33 \mathrm{ng} / \mathrm{ml}$ ).

He was initially admitted in another hospital's Cardiology department with a diagnosis of acute coronary syndrome. At that moment the patient presented with chest pain, ST elevation on ECG, high troponin level, segmental left ventricular hypokinesia, and mid-range ejection fraction. The coronary angiography was normal, and the cardiac MRI confirmed the diagnosis of myocarditis.

Repeated SARS-CoV-2 RT-PCR from nasopharynx and rectal swabs, and rapid antigen testing as well as anti-nucleocapsid $\operatorname{IgG}$ antibodies were negative, while the titre of antispike protein IgG antibodies was high (1342 U/ml). Except for a positive serology for Coxsackie B4 virus (titre >1:640), extensive work-up ruled out viral, bacterial, or parasitic infections, neoplasia, and autoimmune diseases. However, he received broad spectrum antibiotherapy for two weeks.

Following dexamethasone, $16 \mathrm{mg} /$ day iv, the initial clinical course was favourable, symptoms resolved, and cardiac function recovered. When tapering down corticotherapy, symptoms and hyperinflammation reappeared, and iv immunoglobulin $(1 \mathrm{~g} / \mathrm{kg})$ therapy was instituted before the patient was transferred to our department, 19 days after symptom onset.

Coxsackie was not detected by PCR or in cell cultures, from nasopharyngeal and rectal swabs, ruling out viral activity.

There was no significant improvement after oral or iv (3 g methylprednisolone pulse) high-dose corticosteroids, nor after another round of iv immunoglobulins $(2 \mathrm{~g} / \mathrm{kg})$, meaning that every attempt to taper the dose below the equivalent of $100 \mathrm{mg}$ prednisone/day led to a prompt rebound (fever, myalgia, and maculopapular eruption, and increase of neutrophil number, serum CRP, ferritin, procalcitonin, and d-dimers). 

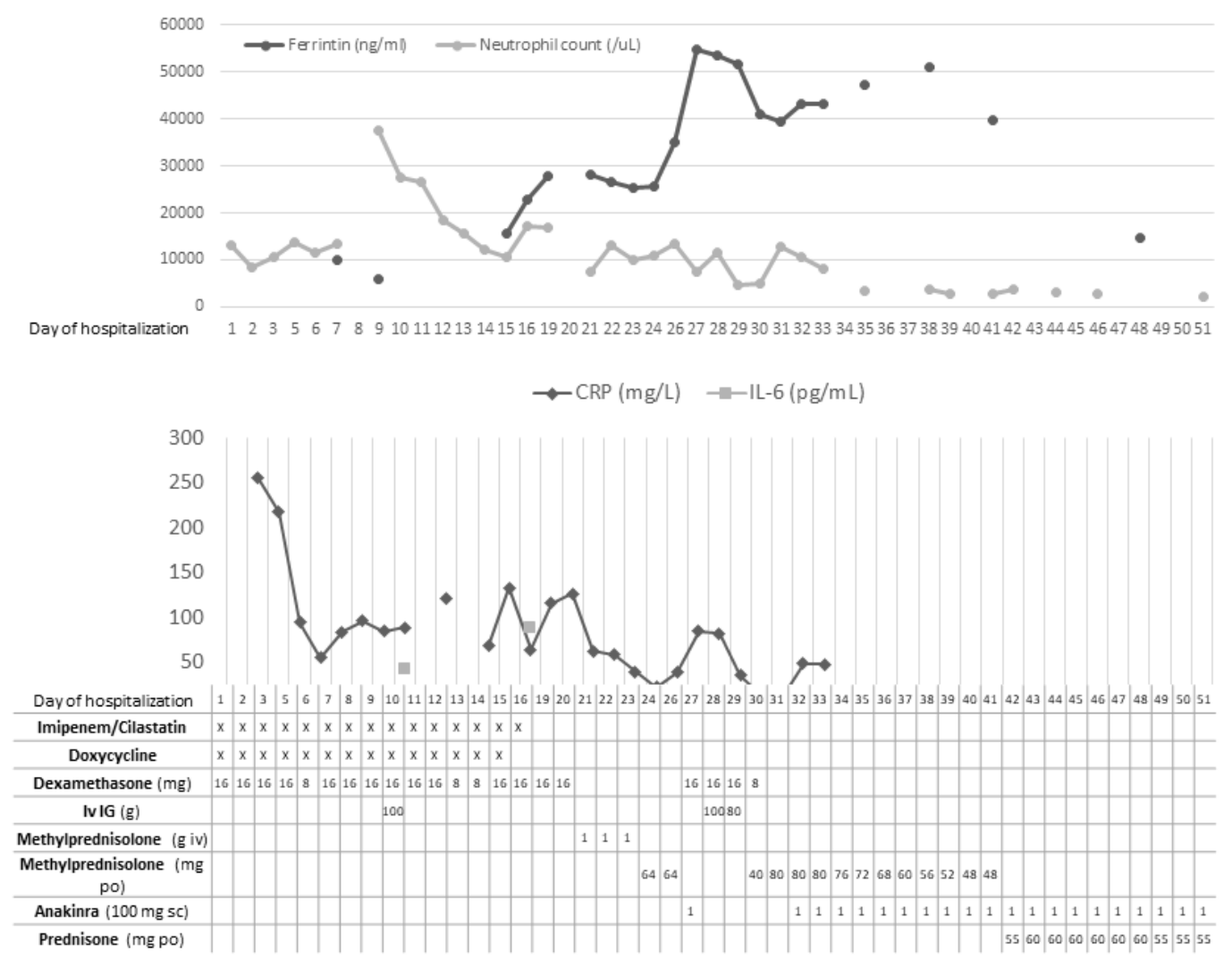

Subsequently, his ferritin levels reached $54,921 \mathrm{ng} / \mathrm{mL}$. On day 32, the patient complained for the first time of sharp arthralgia of both knees, as such, fulfilling the Fautrel's criteria for adultonset Still's disease (AOSD). We decided to initiate anakinra [3], which was highly efficient at the usual dose of $100 \mathrm{mg} /$ day, allowing the rapid tapering of corticosteroids dosage.

The patient was discharged on day 51, after 20 days of anakinra, with significantly lower ferritin levels $(9834 \mathrm{ng} / \mathrm{mL})$ as well as anti-Spike antibodies (617 AU/mL), normal blood cell count, without inflammation and symptom free. Genetic testing identified 5 heterozygous variants of uncertain significance (gene C8A variant c.1334_1336del, gene $\mathrm{C} 8 \mathrm{~A}$ variant c. $1336 \mathrm{~T}>\mathrm{G}$, gene $\mathrm{CARD}$ 9 variant c.464G $>$ A, gene COL7A1 variant c.8017C $>\mathrm{T}$, gene LIG1 variant c. $2014 \mathrm{C}>\mathrm{T}$ ) and no specific mutations for autoinflammatory diseases, HLH or primary immunodeficiency out of an extensive panel.

Since in Romania anakinra was approved only for in-hospital use, for COVID-19 patients, our patient encountered great difficulties in procuring it. This led to intermittent administration, first every other day, then every two days, and finally every three days. When attempted a four day pause, the patient developed arthralgia relapse with fever and sore throat, now fulfilling AOSD
Yamaguchi's criteria, as well (arthralgia lasting $\geq 2$ weeks, all the episodes occurring during flare-ups).

Given the hyperinflammatory state, the initial diagnosis was MIS-A and cytokine storm, considering hemophagocytic lymphohistiocytosis (HLH) and AOSD in the differential diagnosis. According to the Saint-Antoine Hospital score [4], he had a $21 \%$ probability of having HLH; the diagnostic criteria were not met (his soluble IL-2 receptor and triglycerides were high, but he did not develop cytopenia or adeno/splenohepatomegaly).

This patient simultaneously fulfilled the criteria for both AOSD and MIS-A. Although the "game of criteria" would have permitted both diagnoses, the exact terminology is probably less important. AOSD itself is a multisystemic inflammatory syndrome, and the treatment is similar (probably, with the AOSD diagnosis, anakinra would have been introduced earlier). In fact, during the clinical course, we closely assessed for cytopenia, which would have established the diagnosis of macrophage activation syndrome in the case of AOSD, or HLH in the case of postvaccine MIS. However, no cytopenia was noticed.

The first dose of COVID-19 vaccine is a plausible trigger for his hyperinflammatory syndrome, explained by the inflammatory generating potential of Spike proteins [5], the innate and 
adaptive immune mechanisms, including molecular mimicry and the potential immune response mediated by the anti-Spike antibodies [6]. We do not know if the presence of an asymptomatic infection with Coxsackie B4 at the time of vaccination could have had a role, while an eventual Coxsackie infection could have explained only a small part of the features (fever, rash, diarrhoea, and myocarditis).

Regardless of the final formal diagnosis, one of the main particularities of our case was the patient's refractoriness to various therapeutic interventions, including long-term high-dose corticosteroids and intravenous immunoglobulins, but high responsiveness to anakinra. This choice of treatment was determined by the common physiopathological pathways of the considered diagnoses, given the pivotal role of IL-1 in hyperinflammation [7], as well as the potential positive feedback induced by the highly increased ferritin levels, stimulating the macrophages to produce IL1 and therefore even more ferritin [8]. After the first dose of immunization in SARS-Cov-2 naive patients, we identified only two other similar cases: cytokine release syndrome following BTN162b2 vaccination in a 58-year-old male patient with colorectal cancer and long-standing anti-PD-1 monotherapy [9], respectively AOSD after ChAdOx1 vaccination in a 36-year-old male with no comorbities [10].

In conclusion, it is important to recognize the very rare possibility of hyperinflammatory syndromes after COVID-19 immunization and initiate treatment immediately to prevent its life-threatening complications.

Regarding our patient, the ultimate practical question remains: will his disease subside following the decrease of anti-spike protein antibody titre? Or if not, how long will he need to be treated? At this moment, he is well with $100 \mathrm{mg}$ anakinra three times/week.

Correspondence to: Cristian Baicus, M.D, Ph.D, Carol Davila University of Medicine and Pharmacy, Department of Internal Medicine and Cardiology, Colentina University Hospital, Stefan cel Mare Blvd. 19-21, 020125 Bucharest, Romania

E-mail: cristian.baicus@umfcd.ro

\section{REFERENCES}

1. VOGEL TP, TOP KA, KARATZIOS C, et al. Multisystem inflammatory syndrome in children and adults (MIS-C/A): Case definition \& guidelines for data collection, analysis, and presentation of immunization safety data. Vaccine. 2021;39(22):3037.

2. COLAFRANCESCO S, ALESSANDRI C, CONTI F, PRIORI R. COVID-19 gone bad: A new character in the spectrum of the hyperferritinemic syndrome? Autoimmun Rev. 2020;19(7):102573.

3. SALZMAN M, HUANG C-W, O'BRIEN C, CASTILLO R. Multisystem Inflammatory Syndrome after SARS-CoV-2 Infection and COVID-19 Vaccination. Emerg Infect Dis J. 2021;27(7). doi:10.3201/eid2707.210594

4. http://saintantoine.aphp.fr/score/ (last accessed on 28/07/2021)

5. ANGELI F, SPANEVELLO A, REBOLDI G, VISCA D, VERDECCHIA P. SARS-CoV-2 vaccines: Lights and shadows. Eur J Intern Med. 2021;88:1-8.

6. WATAD A, DE MARCO G, MAHAJNA H, et al. Immune-mediated disease flares or new-onset disease in 27 subjects following mrna/dna sars-cov-2 vaccination. Vaccines. 2021;9(5).

7. MEHTA P, CRON RQ, HARTWELL J, MANSON JJ, TATTERSALL RS. Silencing the cytokine storm: the use of intravenous anakinra in haemophagocytic lymphohistiocytosis or macrophage activation syndrome. Lancet Rheumatol. 2020;2(6):e358-e367.

8. CARCILLO JA, KERNAN KK, HORVAT CM, SIMON DW, ANEJA RK. Why and How Is Hyperferritinemic Sepsis Different From Sepsis Without Hyperferritinemia? Pediatr Crit Care Med. 2020;21(5):509.

9. AU L, FENDLER A, SHEPHERD STC, et al. Cytokine release syndrome in a patient with colorectal cancer after vaccination with BNT162b2. Nat Med 2021. May 2021:1-5. doi:10.1038/s41591-021-01387-6

10. LEONE F, CERASUOLO PG, BOSELLO SL, et al. Adult-onset Still's disease following COVID-19 vaccination. Lancet Rheumatol. July 2021. doi:10.1016/s2665-9913(21)00218-6 